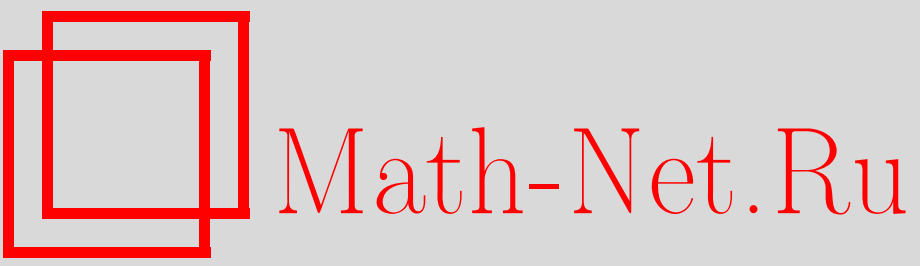

В. М. Бухштабер, Д. В. Лейкин, В. З. Энольский, Матричная реализация гиперэллиптических многообразий Куммера, УМН, 1996, том 51, выпуск 2, 147-148

DOI: https://doi.org/10.4213/rm949

Использование Общероссийского математического портала Math-Net.Ru подразумевает, что вы прочитали и согласны с пользовательским соглашением

http://www.mathnet.ru/rus/agreement

Параметры загрузки:

IP: 34.227 .88 .159

26 апреля 2023 г., 03:15:48 


\title{
МАТРИЧНАЯ РЕАЛИЗАЦИЯ ГИПЕРЭЛЛИПТИЧЕСКИХ МНОГООБРАЗИЙ КУММЕРА
}

\author{
В. М. БУХШТАБЕР, Д. В. ЛЕЙКИН, В.З. ЭНОЛЬСКИЙ
}

Современный интерес к классической проблеме гиперэллиптических якобианов $\operatorname{Jac}(V)$ и их многообразий Куммера $\operatorname{Kum}(V)=\mathrm{Jac} / \pm$ вызван замечательными приложениями в теории интегрируемых систем [1], [2]. Наш подход основан на теории гиперэллиптических функций Клейна [3], [4], в рамках которой удается получить новые реализации многообразий $\operatorname{Jac}(V)$ и $\operatorname{Kum}(V)$ $[5],[6]$.

Рассмотрим пространство $\mathscr{H}$ комплексных симметрических $(g+2) \times(g+2)$-матриц $H=$ $\left\{h_{k, s}\right\}$, у которых $h_{g+2, g+2}=0$ и $h_{g+1, g+2}=2$. Обозначим через $H\left[\begin{array}{l}j_{1}, \ldots, j_{s} \\ i_{1}, \ldots, i_{s}\end{array}\right]$ минор матрицы $H$, соответствующий строкам с номерами $i_{1}, \ldots, i_{s}$ и столбцам с номерами $j_{1}, \ldots, j_{s}$. Сопоставим $H \in \mathscr{H}$ симметрическую $g \times g$-матрицу $A(H)=\left\{a_{k, s}\right\}$, где $a_{k, s}=H\left[\begin{array}{l}k, g+1, g+2 \\ s, g+1, g+2\end{array}\right]$.

Лемма 1. Ранг матриць $H \in \mathscr{H}$ не превосходит 3 тогда и только тогда, когда ранг матрицы $A(H)$ не превосходит 1.

ДокАЗАТЕльство вытекает из тождества Сильвестра (см. [7]): $H\left[\begin{array}{l}i, g+1, g+2 \\ k, g+1, g+2\end{array}\right] H\left[\begin{array}{l}j, g+1, g+2 \\ s, g+1, g+2\end{array}\right]-H\left[\begin{array}{l}i, g+1, g+2 \\ s, g+1, g+2\end{array}\right] H\left[\begin{array}{l}j, g+1, g+2 \\ k, g+1, g+2\end{array}\right]=H\left[\begin{array}{l}i, j, g+1, g+2 \\ k, s, g+1, g+2\end{array}\right] H\left[\begin{array}{l}g+1, g+2 \\ g+1, g+2\end{array}\right]$.

Положим $K \mathscr{H}=\{H \in \mathscr{H}: \operatorname{rank} H \leqslant 3\}$. Для каждой комплексной симметрической $g \times g$-матрицы $A=\left\{a_{k, s}\right\}$ ранга не более 1 существует определенный с точностью до знака $g$-мерный вектор-столбец $Z=Z(A)$ такой, что $A=-4 Z \cdot Z^{T}$, где ${ }^{T}$ - символ транспонирования.

Введем вектора $H_{k}=\left\{h_{k, s} ; s=1, \ldots, g\right\} \in \mathbb{C}^{g}$.

Лемма 2. Отображение

$\gamma: K \mathscr{H} \rightarrow\left(\mathbb{C}^{g} / \pm\right) \times \mathbb{C}^{g} \times \mathbb{C}^{g} \times \mathbb{C}^{1}, \quad \gamma(H)=\left(Z(A(H)), H_{g+1}, H_{g+2}, h_{g+1, g+1}\right)$, является гомеоморфизмом.

ДОКАЗАТЕЛЬСТвО вытекает из соотношения:

$$
4 \widehat{H}=4 Z \cdot Z^{T}+2\left(H_{g+2} H_{g+1}^{T}+H_{g+1} H_{g+2}^{T}\right)-h_{g+1, g+1} H_{g+2} H_{g+2}^{T},
$$

где $\widehat{H}$ - матрица, составленная из векторов-столбцов $H_{k}, k=1, \ldots, g$, а $Z=(Z(A(H)))$.

Введем двулистное разветвленное накрытие $\pi: J \mathscr{H} \rightarrow K \mathscr{H}$, индуцированное накрытием $\mathbb{C}^{g} \rightarrow\left(\mathbb{C}^{g} / \pm\right)$ при помощи отображения $\gamma$.

СледСтвиЕ. $\widehat{\gamma}: J \mathscr{H} \cong \mathbb{C}^{3 g+1}$.

Рассмотрим теперь универсальное пространство $W_{g} g$-х симметрических степеней гиперэллиптических кривых вида

$$
V=\left\{(y, x) \in \mathbb{C}^{2}: y^{2}=4 x^{2 g+1}+\sum_{k=0}^{2 g} \lambda_{2 g-k} x^{2 g-k}\right\}
$$

как алгебраическое подмногообразие в $\left(\mathbb{C}^{2}\right)^{g} \times \mathbb{C}^{2 g+1}$ с координатами

$$
\left\{\left(\left(y_{1}, x_{1}\right), \ldots,\left(y_{g}, x_{g}\right)\right), \lambda_{2 g}, \ldots, \lambda_{0}\right\},
$$

где $\left(\mathbb{C}^{2}\right)^{g}$ есть $g$-я симметрическая степень пространства $\mathbb{C}^{2}$.

Зададим отображение $\lambda: J \mathscr{H} \cong \mathbb{C}^{3 g+1} \rightarrow\left(\mathbb{C}^{2}\right)^{g} \times \mathbb{C}^{2 g+1}$ следующим образом: для $G=$ $\left(Z, H_{g+1}, H_{g+2}, h_{g+1, g+1}\right) \in \mathbb{C}^{3 g+1}$ построим согласно лемме 2 матрицу $\pi(G)=H=\left\{h_{k, s}\right\}$ $\in K \mathscr{H}$ и положим $\lambda(G)=\left\{\left(y_{k}, x_{k}\right), \lambda_{q} ; k=1, \ldots, g, q=0, \ldots, 2 g\right\}$, где $x_{1}, \ldots, x_{g}$ - набор корней уравнения $2 x^{g}+H_{g+2}^{T} X=0$, а $y_{k}=Z^{T} X_{k}, \lambda_{q}=\sum_{i+j=q+2} h_{i, j}$. Здесь $X_{k}=$ $\left(1, x_{k}, \ldots, x_{k}^{g-1}\right)^{T}$.

Работа выполнена при частичной поддержке Российского фонда фундаментальных исследований (грант № 94-01-01444), Международного научного фонда (гранты № M3Z000(ВМБ), № U44000(B3Э)), INTAS 93-1324 (ВЗЭ и ДВЛ). 
Теорема 1. Отображение $\lambda$ индуцирует отображение $Ј \mathscr{H} \cong \mathbb{C}^{3 g+1} \rightarrow W_{g}$.

ДокАЗАтЕльство. Проверка показывает, что имеет место тождество

$$
X_{k}^{T} A X_{s}+4 \sum_{i, j=1}^{g+2} h_{i, j} x_{k}^{i-1} x_{s}^{j-1}=0,
$$

где $A=A(H)$ и $H=\pi(G)$. Полагая $k=s$ и используя соотношение $A=-4 Z \cdot Z^{T}$, получаем $y_{k}^{2}=4 x_{k}^{2 g+1}+\sum_{s=0}^{2 g} \lambda_{2 g-s} x_{k}^{2 g-s}$.

Теперь все готово для описания нашей реализации многообразий

$$
T^{g}=\operatorname{Jac}(V) \text { и } K^{g}=\operatorname{Kum}(V)
$$

для гиперэллиптических кривых в терминах $\sigma$-функций Клейна [3].

Имеем: $\wp p q=-\frac{\partial^{2} \ln \sigma(u)}{\partial u_{p} \partial u_{q}}, \wp_{p q r}=-\frac{\partial^{3} \ln \sigma(u)}{\partial u_{p} \partial u_{q} \partial u_{r}}, p, q, r=1, \ldots, g$, где $u=\left(u_{1}, \ldots, u_{g}\right)$ и $u_{k}=\sum_{j=1}^{g} \int_{x_{0}}^{x_{i}} \frac{x^{k-1} d x}{y}$ (см. [3], [4], а также [5], [6]).

Для каждой неособой кривой $V=\left\{(y, x), y^{2}=4 x^{2 g+1}+\sum_{s=0}^{2 g} \lambda_{2 g-s} x^{2 g-s}\right\}$ зададим следуюшее отображение $\gamma: T^{g} \backslash(\Theta) \rightarrow \mathscr{H}: \gamma(u)=H=\left\{h_{k, s}\right\}$, где $h_{k, s}=4 \wp_{k-1, s-1}-$ $2\left(\wp_{s, k-2}+\wp_{s-2, k}\right)+\frac{1}{2}\left[\delta_{k s}\left(\lambda_{2 s-2}+\lambda_{2 k-2}\right)+\delta_{k+1, s} \lambda_{2 k-1}+\delta_{k, s+1} \lambda_{2 s-1}\right]$. Здесь $(\Theta)-$ тета дивизор и $\delta_{k, s}-$ символ Кронекера.

Tеорема 2. Отображение $\gamma$ индуцирует отображение $T^{g} \backslash(\Theta) \rightarrow$ К $\mathscr{H}$ такое, что $\wp g g k \wp g g s=-\frac{1}{4} a_{k s}(\gamma(u))$, т.е. $\gamma$ поднимается до отображения

$$
\widetilde{\gamma}: T^{g} \backslash(\Theta) \rightarrow J \mathscr{H} \cong \mathbb{C}^{3 g+1} \quad \text { c } \quad Z=(\wp g g 1, \ldots, \wp g g g)^{T} .
$$

Композищия отображений $\lambda \widetilde{\gamma}: T^{g} \backslash(\Theta) \rightarrow W_{g}$ определяет обращение отображения Абеля $A:(V)^{g} \rightarrow T^{g}$ в форме Клейна (см. [3], [5]), и, следовательно, отображение $\widetilde{\gamma}$ является вложением.

Таким образом, получена явная реализация многообразия Куммера $T^{g} \backslash(\Theta) / \pm$ гиперэллиптической кривой $V$ рода $g$ в виде подмногообразия в многообразии матриц $K \mathscr{H}$. Следствием теоремы 2 является, в частности, новое доказательство теоремы Б. А. Дубровина и С.П. Новикова о рациональности универсального пространства якобианов гиперэллиптических кривых $V$ рода $g$ с выделенной точкой ветвления $E_{2 g+2}=\infty$.

\section{СПИСОК ЛИТЕРАТУРЫ}

[1] Дубровин Б.А., Матвеев В.Б., Новиков С.П. // УМН. 1976. Т. 31. № 1. С. 55-135. [2] Мамфорд Д. Лекции о тэта-функциях. М.: Мир, 1988. [3] Klein F. // Math. Ann. 1888. V. 32. P. 351-380. [4] Baker H. F. // Amer. Journ. Math. 1898. V. 20. P. 301-384. [5] Бухштабер В.М., Энольский В.З. // Функц. анализ и его прилож. 1996. Т. 30. №1. [6] Лейкин Д. В. // УМН. 1995. Т. 50. №6. С. 191-192. [7] Хорн Ч., Джонсон Р. Матричный анализ. М.: Мир, 1990. [8] Дубровин Б. А., Новиков С. П. // ДАН СССР. 1974. T. 219. № 3. С. 531-534.

Московский государственный

Принято редколлегией университет им. М. В. Ломоносова, Москва; Институт магнетизма НАН Украины, Киев 